\title{
NORMAL BUNDLES OF LINES ON HYPERSURFACES
}

\author{
HANNAH K. LARSON
}

\begin{abstract}
Let $X \subset \mathbb{P}^{n}$ be a smooth hypersurface. Given a sequence of integers $\vec{a}=\left(a_{1}, \ldots, a_{n-2}\right)$ with $a_{1} \leq \cdots \leq a_{n-2}$, let $F_{\vec{a}}(X)$ be the parameter space of lines $L$ on $X$ such that $N_{L / X} \cong \mathcal{O}\left(a_{1}\right) \oplus \cdots \oplus \mathcal{O}\left(a_{n-2}\right)$. The loci $F_{\vec{a}}(X)$ form a stratification of the Fano scheme of lines on $X$. We show that for general hypersurfaces, the $F_{\vec{a}}(X)$ have the expected dimension and, in this case, compute the class of $\overline{F_{\vec{a}}(X)}$ in the Chow ring of the Grassmannian of lines in $\mathbb{P}^{n}$. For certain splitting types $\vec{a}$, we also provide non-trivial upper bounds on the dimension of $F_{\vec{a}}(X)$ that hold for all smooth $X$.
\end{abstract}

\section{INTRODUCTION}

Let $X \subset \mathbb{P}^{n}$ be a smooth hypersurface of degree $d$ over the complex numbers. This paper is concerned with the geometry of the Fano scheme parameterizing lines on $X$, defined by

$$
F(X):=\{L \in \mathbb{G}(1, n): L \subset X\},
$$

where $\mathbb{G}(1, n)$ is the Grassmannian parameterizing lines in $\mathbb{P}^{n}$. Let $N=\left(\begin{array}{c}n+d \\ d\end{array}\right)-1$ and let $U \subset \mathbb{P}^{N}$ be the open subset parameterizing smooth hypersurfaces of degree $d$ in $\mathbb{P}^{n}$. We define the universal Fano scheme to be

$$
\Sigma:=\{(L, X) \in \mathbb{G}(1, n) \times U: L \subset X\} .
$$

The condition that a hypersurface contain a fixed line is $d+1$ linear conditions on the parameter space $\mathbb{P}^{N}$, so looking at the projection $\Sigma \rightarrow \mathbb{G}(1, n)$, one readily sees that $\Sigma$ is smooth and irreducible of dimension

$$
\operatorname{dim} \Sigma=N-d-1+\operatorname{dim} \mathbb{G}(1, n)=N+2 n-d-3 .
$$

This dimension count gives rise to an expected dimension of $2 n-d-3$ for the Fano scheme of lines on hypersurface of degree $d$ in $\mathbb{P}^{n}$. It is a well-known result that this expected dimension is indeed achieved for general hypersurfaces. A prominent conjecture of Debarre-de Jong states that the expected dimension should be achieved for all smooth hypersurfaces of degree $d \leq n$. The Debarre-de Jong Conjecture has been proved for $d \leq 8$ by Beheshti [1] and for $d \ll n$ by Harris et al. [5].

Here, we study the normal bundles $N_{L / X}$ of lines $L$ in $X$, which govern the local geometry of $F(X)$ at $L$. For each $L \subset X \subset \mathbb{P}^{n}$, there is a short exact 
sequence of normal bundles

$$
\left.0 \rightarrow N_{L / X} \rightarrow N_{L / \mathbb{P}^{n}} \rightarrow N_{X / \mathbb{P}^{n}}\right|_{L} \rightarrow 0,
$$

in which the middle term is $\mathcal{O}(1)^{n-1}$ and the rightmost term is $\mathcal{O}(d)$. Since every vector bundle on $\mathbb{P}^{1}$ splits as a direct sum of line bundles, it follows that

$$
N_{L / X} \cong \mathcal{O}\left(a_{1}\right) \oplus \cdots \oplus \mathcal{O}\left(a_{n-2}\right)
$$

for integers $a_{1} \leq \cdots \leq a_{n-2} \leq 1$ with $a_{1}+\ldots+a_{n-2}=n-d-1$. To study the behavior of the normal bundle, for each sequence of integers $\vec{a}=\left(a_{1}, \ldots, a_{n-2}\right)$ satisfying the above conditions, we define

$$
F_{\vec{a}}(X):=\left\{L \in F(X): N_{L / X} \cong \mathcal{O}\left(a_{1}\right) \oplus \cdots \oplus \mathcal{O}\left(a_{n-2}\right)\right\}
$$

and its universal counterpart

$$
\Sigma_{\vec{a}}:=\left\{(L, X) \in \Sigma: N_{L / X} \cong \mathcal{O}\left(a_{1}\right) \oplus \cdots \oplus \mathcal{O}\left(a_{n-2}\right)\right\}
$$

For convenience of notation, we will abbreviate $\mathcal{O}\left(a_{1}\right) \oplus \cdots \oplus \mathcal{O}\left(a_{n-2}\right)$ by $\mathcal{O}(\vec{a})$.

The loci $\Sigma_{\vec{a}}$ can be realized as the loci where the members of a family of vector bundles on $\mathbb{P}^{1}$ acquire certain splitting types. Let

$$
\Phi:=\left\{(p, L, X) \in \mathbb{P}^{n} \times \mathbb{G}(1, n) \times U: p \in L \subset X\right\}
$$

be the universal line over $\Sigma$ and let $\pi: \Phi \rightarrow \Sigma$ be the projection map. In addition, let

$$
\Psi:=\left\{(p, L, X) \in \mathbb{P}^{n} \times \mathbb{G}(1, n) \times U: p \in X \text { and } L \subset X\right\}
$$

be the universal hypersurface over $\Sigma$. Then, the vector bundle $\mathcal{N}:=N_{\Phi / \Psi}$ has the property that for any $(L, X) \in \Sigma$, the restriction $\left.\mathcal{N}\right|_{\pi^{-1}(L, X)}$ is $N_{L / X}$. It follows that the $\Sigma_{\vec{a}}$ form a stratification of $\Sigma$ with

$$
\overline{\Sigma_{\vec{a}}}=\bigcup_{\vec{a}^{\prime} \leq \vec{a}} \Sigma_{\vec{a}}
$$

where the partial ordering $\leq$ is defined by

$$
\vec{a}^{\prime} \leq \vec{a} \quad \Longleftrightarrow \quad a_{1}^{\prime}+\ldots+a_{k}^{\prime} \leq a_{1}+\ldots+a_{k} \text { for all } k .
$$

See for example Section 14.4.1 of [4]. There is a unique maximal element with respect to this partial ordering, which is determined by the condition $\left|a_{i}-a_{j}\right| \leq 1$ for all $i$ and $j$. We call this the balanced splitting type and all others unbalanced.

In this scenario of a family of vector bundles on $\mathbb{P}^{1}$, deformation theory gives rise to an expected codimension for the loci $\Sigma_{\vec{a}}$. Suppose $\mathcal{E}$ is any family of vector bundles on $\mathbb{P}^{1}$ with base $B$, and let $\pi: \mathbb{P}^{1} \times B \rightarrow B$ be the projection map. For each point $b \in B$ there is an analytic neighborhood $B^{\prime}$ of $b$ and a map from $B^{\prime}$ to the deformation space of the vector bundle $\left.\mathcal{E}\right|_{\pi^{-1}(b)}$ such that $\left.\mathcal{E}\right|_{\pi^{-1}\left(B^{\prime}\right)}$ is equal to the pullback of the versal family on the deformation space. The codimension of the locus of points $b \in B$ where $\left.\mathcal{E}\right|_{\pi^{-1}(b)} \cong \mathcal{O}(\vec{a})$ therefore has codimension at most the dimension of the deformation space of $\mathcal{O}(\vec{a})$. We call this quantity the 
expected codimension for the locus where members acquire splitting type $\vec{a}$ and denote it by

$$
u(\vec{a}):=h^{1}(\mathcal{E} n d(\mathcal{O}(\vec{a})))=\sum_{i<j} \max \left\{a_{j}-a_{i}-1,0\right\} .
$$

Our main results are the following.

Theorem 1.1. $\Sigma_{\vec{a}}$ is smooth and irreducible of codimension $u(\vec{a})$ in $\Sigma$.

Remark. Although the open strata $\Sigma_{\vec{a}}$ are smooth, their closures can and will be singular along the more unbalanced splitting types.

Example 1.2. For degree 7 hypersurfaces in $\mathbb{P}^{5}$, the following diagram indicates which strata lie in the closure of others and the codimension of each strata in $\Sigma$. Here, the balanced splitting type is $(-1,-1,-1)$ at the far right.

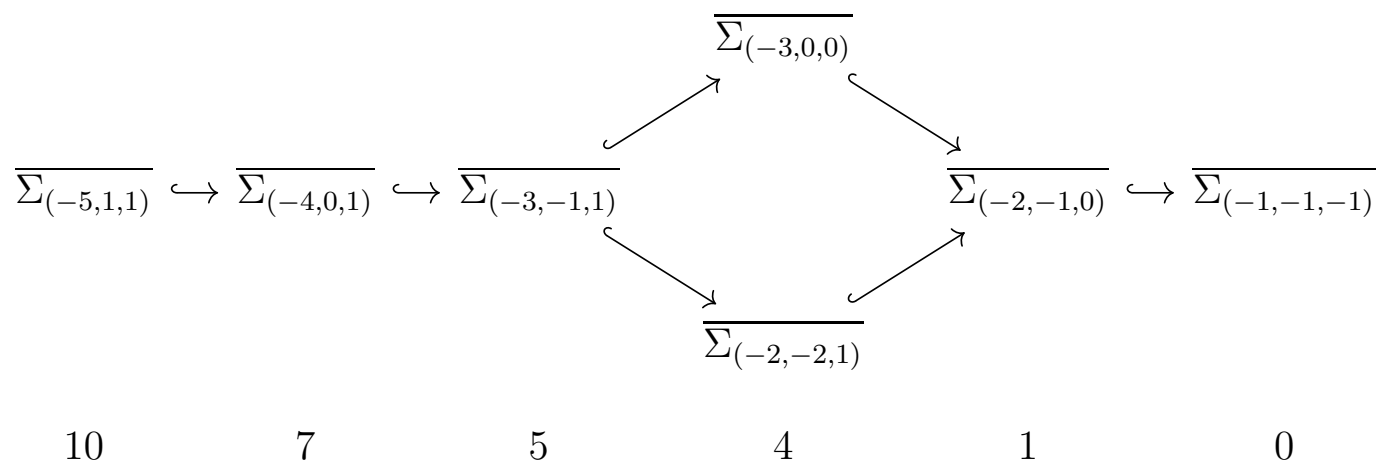

Note that the splitting types $(-3,0,0)$ and $(-2,-2,1)$ cannot specialize to each other, showing that in general the splitting types are not totally ordered.

Theorem 1.3. If $u(\vec{a})>2 n-d-3$ then $F_{\vec{a}}(X)$ is empty for general $X$. If $u(\vec{a}) \leq 2 n-d-3$, then $F_{\vec{a}}(X)$ has codimension $u(\vec{a})$ inside $F(X)$ for general $X$. In this case, the class of $\bar{F}_{\vec{a}}(X)$ in the Chow ring of $\mathbb{G}(1, n)$ is computed by the formula in Proposition 6.1.

Theorem 1.4. If $d \geq 3$, then $\operatorname{dim} F_{(-1,1, \ldots, 1)}(X) \leq n-3$ for all smooth hypersurfaces $X \subset \mathbb{P}^{n}$ of degree $d$. If $d \geq 4$, then $\operatorname{dim} F_{(-a,-b, 1, \ldots, 1)}(X) \leq n-1$ for all smooth hypersurfaces $X \subset \mathbb{P}^{n}$ of degree $d$.

Remark. The questions in this paper could just as well be asked for higher degree rational curves. Recent work of Riedl and Yang [6] shows that when $n \geq d+2$, the locus of rational curves of degree $e$ on general hypersurfaces $X \subset \mathbb{P}^{n}$ of degree $d$ has the "expected codimension" $e(n-d+1)+n-4$. However, the results of Coskun and Riedl in [3] on normal bundles of rational curves in $\mathbb{P}^{n}$ suggest that the normal bundles of higher degree rational curves on hypersurfaces may be less well behaved. 
This paper is organized as follows. In the next section, we put a scheme structure on $\Sigma_{\vec{a}}$ and explain how to compute the class of $\overline{\Sigma_{\vec{a}}}$ for certain splitting types $\vec{a}$, assuming they have the expected codimension. We also provide explicit local equations for $\Sigma_{\vec{a}}$ and describe its functor of points. In Section 3, we prove Theorem 1.1. We then describe the tangent space to $F_{\vec{a}}(X)$ and prove Theorem 1.3 in Section 4. In Section 5, we study singularities on $F_{\vec{a}}(X)$ when $X$ is a cubic threefold and give an important example of the scheme structure of $F_{\vec{a}}(X)$ for $X$ the Fermat quartic threefold. In Section 6 , we find the class of $\overline{F_{\vec{a}}(X)}$ and compute the number of lines with unbalanced normal bundle on a general quintic fourfold as an example. Finally, in Section 7, we prove Theorem 1.4.

Acknowledgements. First and foremost, I would like to thank Professor Joe Harris for all of his encouragement and advice, and meeting with me weekly to discuss ideas. I am also grateful to the 2017 Harvard Program for Research in Science and Engineering (PRISE) and the Herchel Smith Fellowship for their support last summer, when I began working on this project. Finally, thanks to James Hotchkiss for many helpful conversations about this topic and algebraic geometry in general.

\section{THE SCHEME $\Sigma_{\vec{a}}$}

Here, we give $\Sigma_{\vec{a}}$ the structure of a scheme by realizing it as an intersection of loci where certain maps of vector bundles drop rank. We work on the closure of the universal Fano scheme

$$
\bar{\Sigma}:=\left\{(L, X) \in \mathbb{G}(1, n) \times \mathbb{P}^{N}: L \subset X\right\},
$$

and introduce incidence correspondences

$$
\bar{\Phi}:=\left\{(p, L, X) \in \mathbb{P}^{n} \times \mathbb{G}(1, n) \times \mathbb{P}^{N}: p \in L \subset X\right\},
$$

and

$$
\bar{\Psi}:=\left\{(p, L, X) \in \mathbb{P}^{n} \times \mathbb{G}(1, n) \times \mathbb{P}^{N}: p \in X \text { and } L \subset X\right\},
$$

which are just the closures in $\mathbb{P}^{n} \times \mathbb{G}(1, n) \times \mathbb{P}^{N}$ of the varieties defined earlier with the same letters. Next, we set

$$
\mathcal{N}:=N_{\bar{\Phi} / \Psi} \quad \mathcal{E}:=N_{\bar{\Phi} / \mathbb{P}^{n} \times \bar{\Sigma}} \quad \text { and } \quad \mathcal{F}:=\left.N_{\bar{\Psi} / \mathbb{P}^{n} \times \bar{\Sigma}}\right|_{\bar{\Phi}}
$$

so that we have a short exact sequence of sheaves on $\bar{\Phi}$,

$$
0 \longrightarrow \mathcal{N} \longrightarrow \mathcal{E} \stackrel{\phi}{\longrightarrow} \mathcal{F} \longrightarrow 0
$$


Note that $\mathcal{E}$ and $\mathcal{F}$ are vector bundles, but since $\bar{\Psi}$ is singular, $\mathcal{N}$ is not. Let $\mathcal{Q}$ be the universal quotient bundle on $\mathbb{G}(1, n)$. Labeling the relevant projections

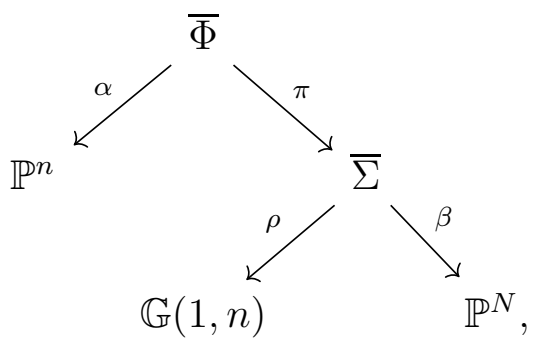

one readily identifies the vector bundles $\mathcal{E}$ and $\mathcal{F}$ as

$$
\mathcal{E}=(\rho \circ \pi)^{*} \mathcal{Q} \otimes \alpha^{*} \mathcal{O}_{\mathbb{P}^{n}}(1) \quad \text { and } \quad \mathcal{F}=\alpha^{*} \mathcal{O}_{\mathbb{P}^{n}}(d) \otimes(\beta \circ \pi)^{*} \mathcal{O}_{\mathbb{P}^{N}}(1) .
$$

For each $i \geq-1$, tensoring (2.1) with $\alpha^{*} \mathcal{O}_{\mathbb{P}^{n}}(i)$ gives rise to a new short exact sequence

$$
0 \longrightarrow \mathcal{N}(i) \longrightarrow \mathcal{E}(i) \stackrel{\phi(i)}{\longrightarrow} \mathcal{F}(i) \longrightarrow 0 \text {. }
$$

Applying pushforward by $\pi$ to the above sequence (2.3), we obtain an exact sequence of sheaves on $\bar{\Sigma}$,

$$
0 \longrightarrow \pi_{*} \mathcal{N}(i) \longrightarrow \pi_{*} \mathcal{E}(i) \stackrel{\pi_{*} \phi(i)}{\longrightarrow} \pi_{*} \mathcal{F}(i) \longrightarrow R^{1} \pi_{*} \mathcal{N}(i) \longrightarrow 0
$$

Since

$$
h^{0}\left(\left.\mathcal{E}(i)\right|_{\pi^{-1}(L, X)}\right)=h^{0}\left(\mathcal{O}_{L}(1+i)^{n-1}\right)=(i+2)(n-1)
$$

and

$$
h^{0}\left(\left.\mathcal{F}(i)\right|_{\pi^{-1}(L, X)}\right)=h^{0}\left(\mathcal{O}_{L}(d+i)\right)=d+i+1
$$

are constant as $(L, X)$ vary over $\bar{\Sigma}$, the theorem on cohomology and base change tells us that $\pi_{*} \mathcal{E}(i)$ and $\pi_{*} \mathcal{F}(i)$ are vector bundles on $\bar{\Sigma}$. Indeed, using the pushpull formula for vector bundles and denoting by $\mathcal{S}^{*}$ the dual of the universal subbundle on $\mathbb{G}(1, n)$, we identify

$$
\pi_{*} \mathcal{E}(i)=\rho^{*} \mathcal{Q} \otimes \rho^{*} \operatorname{Sym}^{1+i} \mathcal{S}^{*} \quad \text { and } \quad \pi_{*} \mathcal{F}(i)=\rho^{*} \operatorname{Sym}^{d+i} \mathcal{S}^{*} \otimes \beta^{*} \mathcal{O}_{\mathbb{P}^{N}}(1) .
$$

Given any map of vector bundles $\psi: E \rightarrow F$ on some base $B$, let $M_{k}(\psi)$ denote the subscheme of $B$ where $\psi$ has rank at most $k$, defined by the $(k+$ $1) \times(k+1)$ minors of $\psi$. Furthermore, let $M_{k}^{\prime}(\psi)$ be the locally closed subscheme $M_{k}(\psi) \backslash M_{k-1}(\psi)$ where $\psi$ has rank exactly $k$. For each $k \geq 0$ and $i \geq-1$, looking at (2.3) , we see that the locus $M_{k}^{\prime}\left(\pi_{*} \phi(i)\right)$ where $\pi_{*} \phi(i)$ has rank exactly $k$ is

$$
M_{k}^{\prime}\left(\pi_{*} \phi(i)\right)=\left\{(L, X) \in \bar{\Sigma}: h^{0}\left(\mathcal{N}(i)_{(L, X)}\right)=(i+2)(n-1)-k\right\} .
$$


Meanwhile, given a splitting type $\vec{a}=\left(a_{1}, \ldots, a_{n-2}\right)$, the locus $\Sigma_{\vec{a}}$ can be described as

$$
\Sigma_{\vec{a}}=\left\{(L, X) \in \Sigma: h^{0}\left(\mathcal{N}(i)_{(L, X)}\right)=t_{i}(\vec{a}) \text { for all } i \geq-1\right\}
$$

where

$$
t_{i}(\vec{a}):=h^{0}(\mathcal{O}(\vec{a}) \otimes \mathcal{O}(i))=\sum_{j=1}^{n-2} \max \left\{0, a_{j}+i+1\right\}
$$

Thus, we can give $\Sigma_{\vec{a}}$ the structure of a scheme by taking an appropriate intersection of schemes $M_{k}^{\prime}\left(\pi_{*} \phi(i)\right)$. For convenience, let

$$
r_{i}(\vec{a}):=(i+2)(n-1)-t_{i}(\vec{a}) .
$$

Definition 2.1. We define the scheme $\Sigma_{\vec{a}}$ as

$$
\Sigma_{\vec{a}}=\Sigma \cap \bigcap_{i=-1}^{d-2} M_{r_{i}(\vec{a})}^{\prime}\left(\pi_{*} \phi(i)\right)
$$

The loci $F_{\vec{a}}(X)$ then inherit a scheme structure as the fibers of $\Sigma_{\vec{a}}$ under projection to the $\mathbb{P}^{N}$ parameterizing hypersurfaces of our given degree and dimension.

Example 2.2 (Cubic hypersurfaces). There are only two splitting types for the normal bundle of a line on a cubic hypersurface: either

$$
N_{L / X}=\mathcal{O}(-1) \oplus \mathcal{O}(1)^{n-3} \quad \text { or } \quad N_{L / X}=\mathcal{O} \oplus \mathcal{O} \oplus \mathcal{O}(1)^{n-4}
$$

Let $\vec{a}=(-1,1, \ldots, 1)$ denote the unbalanced splitting type and $\vec{b}=(0,0,1, \ldots, 1)$ the balanced splitting type. We have

$t_{i}(\vec{a})=\left\{\begin{array}{ll}n-3 & \text { if } i=-1 \\ i+(i+2)(n-3) & \text { if } i \geq 0\end{array} \quad\right.$ and $\quad t_{i}(\vec{b})=i+(i+2)(n-3) \quad \forall i$,

and hence

$r_{i}(\vec{a})=\left\{\begin{array}{ll}2 & \text { if } i=-1 \\ i+4 & \text { if } i \geq 0\end{array} \quad\right.$ and $\quad r_{i}(\vec{b})=i+4 \quad \forall i$.

Since $r_{i}(\vec{a})=r_{i}(\vec{b})$ for all $i \geq 0$, the locus $M_{r_{i}(\vec{a})}^{\prime}\left(\pi_{*} \phi(i)\right)$ is all of $\Sigma$ for these $i$. Therefore, the only non-trivial term in the intersection (2.7) comes from when $i=-1$, giving

$$
\Sigma_{\vec{a}}=\Sigma \cap M_{2}\left(\pi_{*} \phi(-1)\right)
$$


2.1. The class of certain $\overline{\Sigma_{\vec{a}}}$. As seen in the previous example, sometimes $\overline{\Sigma_{\vec{a}}}$ is equal to $M_{k}\left(\pi_{*} \phi(i)\right)$ for some $k$ and $i$. In this case, assuming $\overline{\Sigma_{\vec{a}}}$ has the correct codimension, Porteous' formula will give rise to a formula for the class of $\overline{\Sigma_{\vec{a}}}$ in the Chow ring of $\bar{\Sigma}$.

To set up this formula we need some notation. Given an element $\gamma$ of the Chow ring of a projective scheme $B$, let $\gamma_{i}$ be the component of $\gamma$ in degree $i$. Then, given any $\gamma$ and natural numbers $e$ and $f$, let

$$
\Delta_{f}^{e}(\gamma)=\left(\begin{array}{cccc}
\gamma_{f} & \gamma_{f+1} & \cdots & \gamma_{e+f-1} \\
\gamma_{f-1} & \gamma_{f} & \cdots & \gamma_{e+f-2} \\
\vdots & \vdots & \ddots & \vdots \\
\gamma_{f-e+1} & \gamma_{f-e+2} & \cdots & \gamma_{f}
\end{array}\right)
$$

In general, Porteous' formula says that if $\psi: E \rightarrow F$ is any map of vector bundles of ranks $e$ and $f$ on $B$ and the scheme $M_{k}(\psi)$ has codimension $(f-k)(e-k)$ in $B$, then the class of $M_{k}(\phi)$ in the Chow ring of $B$ is

$$
\left[M_{k}(\phi)\right]=\Delta_{f-k}^{e-k}\left(\frac{c(F)}{c(E)}\right)
$$

where $c(F)$ and $c(E)$ are the total Chern classes of $F$ and $E$.

The $\vec{a}$ for which we will calculate the class of $\overline{\Sigma_{\vec{a}}}$ have the form

$$
\vec{a}=(s_{1}, \ldots, s_{n-2-m}, \underbrace{1, \ldots, 1}_{m}) \quad \text { where } \quad \sum_{i<j} \max \left\{0, s_{j}-s_{i}-1\right\}=0 .
$$

The closure of such $\Sigma_{\vec{a}}$ in $\Sigma$ consists of pairs $(L, X)$ where $N_{L / X}$ has at least $m$ copies of $\mathcal{O}(1)$ in it, whose closure in $\bar{\Sigma}$ is exactly $M_{r_{-1}(\vec{a})}\left(\pi_{*} \phi(-1)\right)$. In addition, for such $\vec{a}$, we always have

$$
u(\vec{a})=m b=\left(\operatorname{rank}\left(\pi_{*} \mathcal{E}(-1)\right)-r_{-1}(\vec{a})\right)\left(\operatorname{rank}\left(\pi_{*} \mathcal{F}(-1)\right)-r_{-1}(\vec{a})\right)
$$

where $b=m-(n-d-1)$. Applying Porteous' formula, we immediately arrive at the following.

Proposition 2.3. Suppose $\vec{a}$ has the form (2.9), and set $b=m-(n-d-1)$. If $\overline{\Sigma_{\vec{a}}}$ has the expected codimension $u(\vec{a})$ in $\bar{\Sigma}$, then its class in the Chow ring of $\bar{\Sigma}$ is given by

$$
\left[\overline{\Sigma_{\vec{a}}}\right]=\Delta_{b}^{m}\left(\frac{c\left(\pi_{*} \mathcal{F}(-1)\right)}{c\left(\pi_{*} \mathcal{E}(-1)\right)}\right) .
$$

Remark. Given that each $\Sigma_{\vec{a}}$ has the "expected codimension", it is natural to wonder if a similar formula can be found for all $\vec{a}$. One obstacle to following the same approach as above is that for $i>-1$, the loci $M_{r_{i}(\vec{a})}\left(\pi_{*} \phi(i)\right)$ pick up 
components of larger dimension. For example, when $n=6$ and $d=11$, so that the rank of the normal bundle is 4 and its degree is -6 , we have

$$
M_{2}\left(\pi_{*} \phi(0)\right)=\overline{\Sigma_{(-3,-3,0,0)}} \cup \overline{\Sigma_{(-3,-2,-2,1)}} .
$$

To use Proposition 2.3 in practice, we need to describe the Chow ring of $\bar{\Sigma}$ and find the Chern classes of $\pi_{*} \mathcal{E}(i)$ and $\pi_{*} \mathcal{F}(i)$. This is easily done since $\bar{\Sigma}$ is a projective bundle over $\mathbb{G}(1, n)$ and the vector bundles $\pi_{*} \mathcal{E}(i)$ and $\pi_{*} \mathcal{F}(i)$ are tensor products of vector bundles with known Chern classes, and hence their Chern classes are determined by the splitting principle.

2.2. Explicit local equations. For each line $L_{0} \in \mathbb{G}(1, n)$, we can choose coordinates on $\mathbb{P}^{n}$ so that $L_{0}=V\left(x_{2}, \ldots, x_{n}\right)$ and affine coordinates $a_{i j}$ on $\mathbb{G}(1, n)$ where

$$
\left(\begin{array}{llll}
a_{20} & a_{30} & \cdots & a_{n 0} \\
a_{21} & a_{31} & \cdots & a_{n 1}
\end{array}\right) \quad \longleftrightarrow \quad L: x_{i}=a_{i 0} x_{0}+a_{i 1} x_{1} .
$$

Over the product of this affine open with $\mathbb{P}^{N}$, the map $\phi$ in (2.1) is given by $n-1$ homogeneous polynomials of degree $d-1$,

$$
\frac{\partial f}{\partial x_{i}}\left(x_{0}, x_{1}, a_{20} x_{0}+a_{21} x_{1}, \ldots, a_{n 0} x_{0}+a_{n 1} x_{1}\right)=C_{i 0} x_{0}^{d-1}+\ldots+C_{i d-1} x_{1}^{d-1}
$$

where the $C_{i j}$ are bihomogeneous polynomials in the $a_{k \ell}$ and the coefficients of $f$, which are our coordinates on $\mathbb{P}^{N}$.

Over this open subset, the vector bundle $\pi_{*} \mathcal{E}(i)$ is naturally identified with the trivial bundle with fiber $H^{0}\left(\mathcal{O}_{L_{0}}(1+i)\right)^{n-1}$ and similarly $\pi_{*} \mathcal{F}(i)$ is naturally identified with $H^{0}\left(\mathcal{O}_{L_{0}}(d+i)\right) \otimes \beta^{*} \mathcal{O}_{\mathbb{P}^{N}}(1)$. In terms of the standard basis of monomials on each copy of $H^{0}(\mathcal{O}(1+i))$ and $H^{0}(\mathcal{O}(d+i))$, the map $\pi_{*} \phi(i)$ is represented by the matrix

$$
C(i)=\left(\begin{array}{ccccccccc}
C_{2,0} & 0 & \cdots & 0 & \ldots & C_{n, 0} & 0 & \cdots & 0 \\
C_{2,1} & C_{2,0} & \cdots & 0 & \cdots & C_{n, 1} & C_{n, 0} & \cdots & 0 \\
\vdots & C_{2,1} & \ddots & \vdots & \ldots & \vdots & C_{n, 1} & \ddots & \vdots \\
C_{2, d-1} & \vdots & \ddots & C_{2,0} & \cdots & C_{n, d-1} & \vdots & \ddots & C_{n, 0} \\
0 & C_{2, d-1} & & C_{2,1} & \cdots & 0 & C_{n, d-1} & & C_{n, 1} \\
\vdots & \vdots & \ddots & \vdots & \cdots & \vdots & \vdots & \ddots & \vdots \\
0 & 0 & \cdots & C_{2, d-1} & \cdots & 0 & 0 & \cdots & C_{n, d-1}
\end{array}\right)
$$

The local equations for $M_{r_{i}(\vec{a})}\left(\pi_{*} \phi(i)\right)$ are exactly the $\left(r_{i}(\vec{a})+1\right) \times\left(r_{i}(\vec{a})+1\right)$ minors of this matrix. The collection of all these minors as $i$ runs from -1 to $d-2$ are thus local equations for $\overline{\Sigma_{\vec{a}}}$. 
Example $2.4(n=4, d=3)$. We have $\Sigma_{\vec{a}}=M_{2}\left(\pi_{*} \phi(-1)\right)$, which is defined by the single equation

$$
\operatorname{det} C(-1)=\operatorname{det}\left(\begin{array}{lll}
C_{2,0} & C_{3,0} & C_{4,0} \\
C_{2,1} & C_{3,1} & C_{4,1} \\
C_{2,2} & C_{3,2} & C_{4,2}
\end{array}\right) .
$$

Since this equation is non-zero, this provides a direct proof that $\Sigma_{\vec{a}} \subset \Sigma$ has codimension 1, which is the expected codimension for this locus.

2.3. The functor of points. As one might hope, $\Sigma_{\vec{a}}$ has a nice description in terms of its functor of points. Given any morphism $\eta: Z \rightarrow \Sigma$, one obtains a diagram

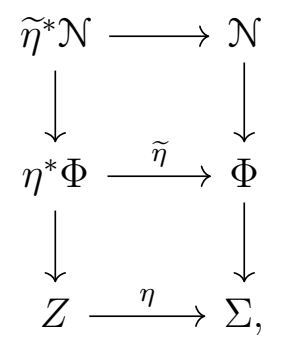

where $\widetilde{\eta}^{*} \mathcal{N}$ is a family of vector bundles on $\mathbb{P}^{1}$ with base $Z$. We say that such a family has constant local splitting type $\vec{a}$ if $Z$ can be covered by open sets $U$ over which $\left(\eta^{*} \Phi\right)_{U} \cong U \times \mathbb{P}^{1}$ and

$$
\left(\widetilde{\eta}^{*} \mathcal{N}\right)_{U} \cong \alpha^{*} \mathcal{O}(\vec{a})
$$

where $\alpha:\left(\eta^{*} \Phi\right)_{U} \cong U \times \mathbb{P}^{1} \rightarrow \mathbb{P}^{1}$ is the projection map. With this notion, we have the following.

Lemma 2.5. Let $\mathcal{N}=N_{\Phi / \Psi}$ as before. Then

$$
\operatorname{Mor}\left(Z, \Sigma_{\vec{a}}\right)=\left\{\eta \in \operatorname{Mor}(Z, \Sigma): \widetilde{\eta}^{*} \mathcal{N} \text { has constant local splitting type } \vec{a}\right\} \text {. }
$$

Proof. First we check that the restriction of $\mathcal{N}$ to $\Sigma_{\vec{a}}$ has constant local splitting type $\vec{a}$. For each $(L, X) \in \Sigma_{\vec{a}}$, let $U \subset \Sigma_{\vec{a}}$ be an open set containing $(L, X)$ over which $\mathcal{E}_{U}$ can be trivialized, i.e.

$$
\mathcal{E}_{U} \cong H^{0}\left(\mathcal{O}_{L}(1)^{n-1}\right) \otimes \mathcal{O}_{U}
$$

By the theorem on cohomology and base change, $\pi_{*} \mathcal{N}(i)$ is a vector bundle on $\Sigma_{\vec{a}}$. After passing to a possibly smaller open set $U$, we can assume that

$$
\pi_{*} \mathcal{N}(i)_{U}=\operatorname{ker} \pi_{*} \phi(i)_{U}: \pi_{*} \mathcal{E}(i)_{U} \rightarrow \pi_{*} \mathcal{F}(i)_{U}
$$

can be trivialized for all $i=-1, \ldots, d-2$. An inclusion of $\alpha^{*} \mathcal{O}\left(a_{k}\right)$ into the vector bundle $\mathcal{E}_{U} \cong H^{0}\left(\mathcal{O}_{L}(1)^{n-1}\right) \otimes \mathcal{O}_{U}$ is specified by a collection of $n-1$ polynomials of degree $1-a_{k}$ in $x_{0}, x_{1}$ with coefficients rational functions on $U$ that do not simultaneously vanish, i.e. a non-zero section of

$$
H^{0}\left(\mathcal{O}_{L}\left(1-a_{k}\right)^{n-1}\right) \otimes \mathcal{O}_{U}=\pi_{*} \mathcal{E}\left(-a_{k}\right)_{U}
$$


Such a section will define an inclusion of $\alpha^{*} \mathcal{O}\left(a_{k}\right)$ into $\mathcal{N}_{U}$ exactly when the section lies in the kernel of $\pi_{*} \phi\left(-a_{k}\right)_{U}$.

Now let $m_{i}$ be the number of $i$ 's in the list $\vec{a}$. Suppose we have a local frame of the kernel of $\pi_{*} \phi(-1)_{U}$,

$$
w^{(k)}=\left(w_{2}^{(k)}, \ldots, w_{n}^{(k)}\right) \in H^{0}\left(\mathcal{O}_{L}^{n-1}\right) \otimes \mathcal{O}_{U}=\mathcal{O}_{U}^{n-1} \quad k=1, \ldots, m_{1} .
$$

We observe that the elements

$$
\left(w_{2}^{(k)} x_{0}, \ldots, w_{n}^{(k)} x_{0}\right) \text { and }\left(w_{2}^{(k)} x_{1}, \ldots, w_{n}^{(k)} x_{1}\right) \in H^{0}(\mathcal{O}(1))^{n-1} \otimes \mathcal{O}_{U}
$$

are in the kernel of $\pi_{*} \phi_{U}$. Since ker $\pi_{*} \phi_{U}=\pi_{*} \mathcal{N}_{U}$ has rank $2 m_{1}+m_{0}$, we can find another $m_{0}$ independent sections of $H^{0}\left(\mathcal{O}(1)^{n-1}\right) \otimes \mathcal{O}_{U}$ in the kernel of $\pi_{*} \phi_{U}$. These $m_{0}$ sections define an inclusion $\alpha^{*} \mathcal{O}^{m_{0}} \rightarrow \mathcal{N}$ which is independent from our previous inclusion of $\alpha^{*} \mathcal{O}(1)^{m_{1}}$.

Continuing in this way, the conditions on the rank of $\pi_{*} \phi(i)$ guarantee that we can find sections in its kernel that define inclusions of $\alpha^{*} \mathcal{O}(-i)^{m_{-i}}$ independent from those defined before for lower $i$. Hence, the restriction of $\mathcal{N}$ to $\Sigma_{\vec{a}}$ has constant local splitting type $\vec{a}$.

It remains to show that if $\eta: Z \rightarrow \Sigma$ is such that $\widetilde{\eta}^{*} \mathcal{N}$ has constant local splitting type $\vec{a}$ then $\eta$ factors through $\Sigma_{\vec{a}}$. If the restriction of $\widetilde{\eta}^{*} \mathcal{N}$ to some $U \subset Z$ is $\alpha^{*} \mathcal{O}(\vec{a})$, then the $\left(r_{i}(\vec{a})+1\right) \times\left(r_{i}(\vec{a})+1\right)$ minors of $\widetilde{\eta}^{*} \phi(i)$ vanish. But this is given locally by $\eta^{\#}$ applied to the minors of $C(i)$, where $\eta^{\#}: \mathcal{O}_{\Sigma} \rightarrow \mathcal{O}_{Z}$ is the map of structure sheaves. Thus, $\eta^{\#}$ kills the ideal of $\Sigma_{\vec{a}}$, which is to say $\eta$ factors through the subscheme $\Sigma_{\vec{a}} \subset \Sigma$.

\section{Proof of Theorem 1.1}

In this section, we prove Theorem 1.1, providing two proofs of the dimension statement. The first uses a standard dimension-counting argument with an incidence correspondence and also proves smoothness and irreducibility. The second uses deformation theory to obtain the dimension statement directly. Recall the statement of the theorem.

Theorem 3.1. $\Sigma_{\vec{a}}$ is smooth and irreducible of codimension $u(\vec{a})$ in $\Sigma$.

Proof. First note that, like the universal Fano scheme, the projection onto the second factor $\rho_{\vec{a}}: \Sigma_{\vec{a}} \rightarrow \mathbb{G}(1, n)$ is a fiber bundle. Thus, to prove the theorem, it will suffice to prove that the fibers of $\rho_{\vec{a}}: \Sigma_{\vec{a}} \rightarrow \mathbb{G}(1, n)$ are smooth and irreducible of codimension $u(\vec{a})$ in the fibers of the projection of the universal Fano scheme onto its second factor, $\rho: \Sigma \rightarrow \mathbb{G}(1, n)$.

So, fix a line $L=V\left(x_{2}, \ldots, x_{n}\right)$ and let

$$
\Omega=\left\{(X, \varphi) \in U \times \operatorname{Aut}(\mathcal{O}(\vec{a})): X \supset L \text { and } \varphi: \mathcal{O}(\vec{a}) \cong N_{L / X}\right\},
$$

where $U \subset \mathbb{P}^{N}$ is the open subset parameterizing smooth hypersurfaces. First consider the projection $\alpha: \Omega \rightarrow U$. The image of $\alpha$ is exactly $\rho_{\vec{a}}^{-1}(L)$, and $\alpha$ makes $\Omega$ into a fiber bundle over its image with smooth fibers isomorphic to 
Aut $(\mathcal{O}(\vec{a}))$. Irreducibility and smoothness of $\rho_{\vec{a}}^{-1}(L)$ will thus follow from the same properties of $\Omega$.

Next, we construct a map $\beta: \Omega \rightarrow \operatorname{Hom}\left(\mathcal{O}(\vec{a}), \mathcal{O}(1)^{n-1}\right)$ by setting $\beta((X, \varphi))$ to be the composition

$$
\varphi: \mathcal{O}(\vec{a}) \cong N_{L / X} \longrightarrow N_{L / X} \cong \mathcal{O}(1)^{n-1} .
$$

The image of $\beta$ is certainly contained in the open subset of injective maps, and we claim that all injective maps are in the image. Indeed, given any

$$
0 \longrightarrow \mathcal{O}(\vec{a}) \stackrel{A}{\longrightarrow} \mathcal{O}(1)^{n-1},
$$

the cokernel is a line bundle of degree $d$, so we have a short exact sequence

$$
0 \longrightarrow \mathcal{O}(\vec{a}) \stackrel{A}{\rightarrow} \mathcal{O}(1)^{n-1} \stackrel{\left(m_{1}, \ldots, m_{n-1}\right)}{\longrightarrow} \mathcal{O}(d) \longrightarrow 0 .
$$

Explicitly, if we think of $A$ as being represented by an $(n-1) \times(n-2)$ matrix with polynomials of degree $1-a_{j}$ in the $j$ th column, then the $m_{i}$ are the maximal minors of $A$. We claim that

$$
\beta^{-1}(A)=\left\{(V(f), \varphi): f=\sum_{i=2}^{n} x_{i} m_{i+1}+\left(x_{2}, \ldots, x_{n}\right)^{2} \text { smooth }\right\} .
$$

If $X=V(f)$ is any hypersurface containing $L$, then we have a short exact sequence

$$
0 \rightarrow N_{L / X} \stackrel{\iota}{\rightarrow} \mathcal{O}_{L}(1)^{n-1} \stackrel{\left(f_{2}, \ldots, f_{n}\right)}{\longrightarrow} \mathcal{O}_{L}(d) \rightarrow 0,
$$

where $f_{i}=\left.\frac{\partial f}{\partial x_{i}}\right|_{L}$. So for $(X, \varphi)$ to be in $\beta^{-1}(A)$, we must have $f_{i}=m_{i+1}$, and hence $f$ has the claimed form. On the other hand, whenever $f$ has this form, the right hand maps in (3.1) and (3.3) are equal, so by the universal property of kernel there exists a unique isomorphism of their kernels $\varphi: \mathcal{O}(\vec{a}) \cong N_{L / X}$ such that $\varphi \circ \iota=A$, implying $\beta((V(f), \varphi))=A$.

Next, observe that the $m_{i}$ have no common zeros, so every $f$ of the form in (3.2) is smooth along $L$. Thus, Bertini's theorem tells us that the general such $f$ is smooth. That is, $\beta^{-1}(A)$ is an open dense subset of this linear system. In particular, $\beta^{-1}(A)$ is smooth and irreducible of dimension

$$
\operatorname{dim} \beta^{-1}(A)=(N+1)-d-1-(n-1) d .
$$

Our assumption that $a_{i} \leq 1$ for all $i$ guarantees that the image $\beta(\Omega)$, which is equal to the injective maps, is an open dense subset of $H^{0}\left(\mathcal{H} \circ m\left(\mathcal{O}(\vec{a}), \mathcal{O}(1)^{n-1}\right)\right)$. It follows that $\Omega$ is smooth and irreducible of dimension

$$
\operatorname{dim} \Omega=h^{0}(\mathcal{H} \operatorname{com}(\mathcal{O}(\vec{a}), \mathcal{O}(1)))+(N+1)-d-1-(n-1) d .
$$

Finally, since the fibers of $\alpha$ are copies of $\operatorname{Aut}(\mathcal{O}(\vec{a}))$, we have $\operatorname{dim} \alpha(\Omega)=h^{0}(\mathcal{H} \operatorname{om}(\mathcal{O}(\vec{a}), \mathcal{O}(1)))+(N+1)-d-1-(n-1) d-h^{0}(\mathcal{E} n d(\mathcal{O}(\vec{a})))$. 
Hence,

$$
\begin{aligned}
\operatorname{codim}\left(\rho_{\vec{a}}^{-1}(L) \subset \rho^{-1}(L)\right)= & N-d-1-\operatorname{dim} \alpha(\Omega) \\
=d(n-1)-1- & h^{0}\left(\mathcal{H} \operatorname{com}\left(\mathcal{O}(\vec{a}), \mathcal{O}_{L}(1)^{n-1}\right)\right) \\
& +h^{0}(\mathcal{E} n d(\mathcal{O}(\vec{a}))) .
\end{aligned}
$$

Next, observe that

$$
\begin{aligned}
h^{0}(\mathcal{H o m}(\mathcal{O}(\vec{a}), \mathcal{O}(d))) & =\sum_{i=1}^{n-2} h^{0}\left(\mathcal{O}\left(d-a_{i}\right)\right)=\sum_{i=1}^{n-2}\left(d-a_{i}+1\right) \\
& =(d+1)(n-2)-(n-d-1) \\
& =d(n-1)-1 .
\end{aligned}
$$

After substituting this in above, applying $\mathcal{H} \operatorname{om}(\mathcal{O}(\vec{a}),-)$ to the short exact sequence

$$
0 \longrightarrow \mathcal{O}(\vec{a}) \longrightarrow \mathcal{O}_{L}(1)^{n-1} \longrightarrow \mathcal{O}(d) \longrightarrow 0,
$$

and setting the alternating sum of the dimension of terms in the long exact sequence in cohomology to zero, we see that

$$
\operatorname{codim}\left(\rho_{\vec{a}}^{-1}(L) \subset \rho^{-1}(L)\right)=h^{1}(\mathcal{E} n d(\mathcal{O}(\vec{a})))=u(\vec{a}),
$$

as desired.

Given that $\Sigma_{\vec{a}}$ has the expected codimension $h^{1}(\mathcal{E} n d(\mathcal{O}(\vec{a})))$ coming from deformation theory, one might wonder if there is a direct proof of this fact using deformation theory. This can be carried out as follows.

Alternative proof of dimension count. For each point $(L, X) \in \Sigma$, there exists an analytic neighborhood $B$ of $(L, X)$ and a map $\phi: B \rightarrow \Delta=\operatorname{Def}\left(N_{L / X}\right)$ so that

$$
\left.\mathcal{N}\right|_{\pi^{-1}(B)} \cong \phi^{*} \mathcal{F}
$$

where $\mathcal{F} \rightarrow \Delta \times \mathbb{P}^{1}$ is the miniversal family. The claim will follow from showing that the differential of $\phi$ is surjective at $(L, X)$. To do this, we construct a set $B^{\prime} \subset B$ containing $(L, X)$ which lifts an open neighborhood of the distinguished point $0 \in \Delta$.

Consider the projections

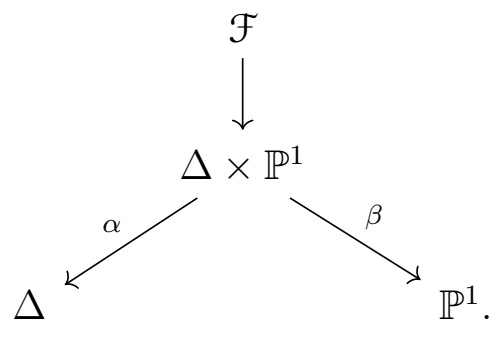


First, we will show that there exists a neighborhood $V$ of $0 \in \Delta$ so that $\left.\mathcal{F}\right|_{\alpha^{-1}(V)}$ admits an inclusion into the trivial family $\beta^{*} \mathcal{O}(1)^{n-1}$. This is equivalent to finding a local section of $\alpha_{*} \mathcal{H} \operatorname{om}\left(\mathcal{F}, \beta^{*} \mathcal{O}(1)^{n-1}\right)$ which is corresponds to an injective map on each fiber. Since each subbline bundle of $\mathcal{F}_{\delta}$ for $\delta \in \Delta$ has degree at most 1 , we have

$$
h^{0}\left(\mathcal{F}_{\delta}, \beta^{*} \mathcal{O}(1)^{n-1}\right)=(n-1) \sum_{i=1}^{n-2}\left(2-a_{i}(\delta)\right)=(n-1)(2(n-2)-n-d-1),
$$

which is constant as $\delta$ varies over $\Delta$. Hence, the theorem on cohomology and base change tells us that $\alpha_{*} \mathcal{H} \operatorname{Com}\left(\mathcal{E}, \beta^{*} \mathcal{O}(1)^{n-1}\right)$ is a vector bundle. Let $\iota$ be the inclusion

$$
\iota: N_{L / X} \rightarrow N_{L / \mathbb{P}^{n}} \cong \mathcal{O}(1)^{n-1} .
$$

Since the rank of a family of maps drops on closed subsets, we can find a local section of $\alpha_{*} \mathcal{H} \operatorname{om}\left(\mathcal{F}, \beta^{*} \mathcal{O}(1)^{n-1}\right)$ which is $\iota$ over the origin and injective on each fiber $\mathcal{F}_{\delta}$ for $\delta$ in some neighborhood $V$.

This gives rise to a short exact sequence

$$
\left.0 \rightarrow \mathcal{F}\right|_{\alpha^{-1}(V)} \rightarrow \beta^{*} \mathcal{O}_{\mathbb{P}^{1}}(1)^{n-1} \rightarrow \mathcal{L} \rightarrow 0
$$

where $\mathcal{L}$ is a line bundle that restricts to $\mathcal{O}_{\mathbb{P}^{1}}(d)$ on each fiber of $\alpha$. For each $v \in V$, the map $\beta^{*} \mathcal{O}_{\mathbb{P}^{1}}(1)^{n-1} \rightarrow \mathcal{L}$ restricted to $\alpha^{-1}(v)$ is given by a collection of $n-1$ homogeneous polynomials $g_{2}\left(v ; x_{0}, x_{1}\right), \ldots, g_{n}\left(v ; x_{0}, x_{1}\right)$ of degree $d-1$. Fixing coordinates so that $L=V\left(x_{2}, \ldots, x_{n}\right)$, if $f$ is the defining equation of $X$, then

$$
g_{i}\left(0 ; x_{0}, x_{1}\right)=\left.\frac{\partial f}{\partial x_{i}}\right|_{L}
$$

Next, let

$$
\tilde{f}=f-\left.\sum_{i=2}^{n} x_{i} \frac{\partial f}{\partial x_{i}}\right|_{L} \in\left(x_{2}, \ldots, x_{n}\right)^{2}
$$

and consider the polynoimals

$$
f(v)=\sum_{i=2}^{n} x_{i} g_{i}\left(v ; x_{0}, x_{1}\right)+\widetilde{f}
$$

Note that all of the $f(v)$ vanish on $L$ and we have $f(0)=f$, which is smooth. Since singular hypersurfaces are a closed subset, after restricting to a possibly smaller neighborhood $0 \in V^{\prime} \subset V$, we will have $f(v)$ smooth for all $v \in V^{\prime}$. In particular, the collection of hypersurfaces defined by $f(v)$ for $v \in V^{\prime}$ is a slice around $(L, X)$ that $\phi$ maps one-to-one onto $V^{\prime}$. 


\section{The Dimension of $F_{\vec{a}}(X)$ IN General}

In this section, we determine the dimension of $F_{\vec{a}}(X)$ for general $X$. It follows immediately from Theorem 1.1 that if $u(\vec{a})>2 n-d-3$, then $F_{\vec{a}}(X)$ is empty for general $X$. On the other hand, if $u(\vec{a}) \leq 2 n-d-3$, then provided $\Sigma_{\vec{a}}$ dominates $U \subset \mathbb{P}^{N}$, it follows that $\operatorname{codim}\left(F_{\vec{a}}(X) \subset F(X)\right)=u(\vec{a})$ for general $X$. Moreover, by upper semicontinuity of the dimension of fibers of $\Sigma_{\vec{a}} \rightarrow U \subset \mathbb{P}^{N}$, to prove this holds, it suffices to find some $L \subset X$ such that $\operatorname{dim} T_{L} F_{\vec{a}}(X)=2 n-d-3-u(\vec{a})$. In the following, we give an explicit description of the tangent space to $F_{\vec{a}}(X)$, and then exhibit such an $L \subset X$.

Let $P$ be the incidence correspondence

$$
P=\{(p, L) \in X \times F(X): p \in L\},
$$

and let $T=\operatorname{Spec} \mathbb{C}[\epsilon] /\left(\epsilon^{2}\right)$. Every $v: T \rightarrow F(X)$ in $\operatorname{Mor}_{L}(T, F(X))=T_{L} F(X)$ gives rise to a diagram

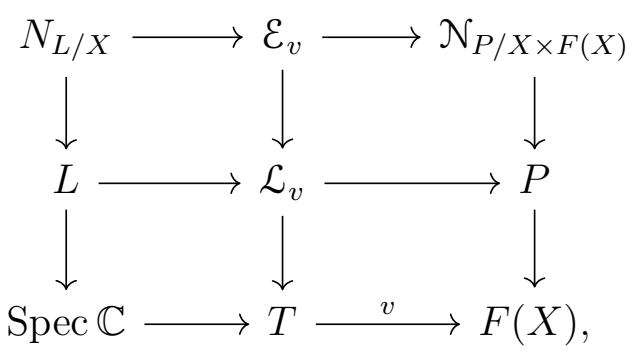

where $\mathcal{E}_{v}$ is a first order deformation of the vector bundle $N_{L / X}$. Given such an $\mathcal{L}_{v} \cong T \times \mathbb{P}^{1}$, let $\beta: \mathcal{L}_{v} \rightarrow \mathbb{P}^{1}$ be the projection map onto the second factor.

Lemma 4.1. The tangent vector $v: T \rightarrow F(X)$ in $T_{L} F(X)$ is in the subspace $T_{L} F_{\vec{a}}(X)$ if and only if the associated first order deformation $\mathcal{E}_{v}$ of $N_{L / X}$ is trivial, meaning

$$
\mathcal{E}_{v} \cong \beta^{*} \mathcal{O}_{\mathbb{P}^{1}}\left(a_{1}\right) \oplus \ldots \oplus \beta^{*} \mathcal{O}_{\mathbb{P}^{1}}\left(a_{n-2}\right) .
$$

Proof. Restricting our description of the functor of points for $\Sigma_{\vec{a}}$ to the fiber of $\Sigma_{\vec{a}} \rightarrow U \subset \mathbb{P}^{N}$ over $X$, we see that

$$
\begin{aligned}
& \operatorname{Mor}\left(T, F_{\vec{a}}(X)\right) \\
& =\left\{f \in \operatorname{Mor}(T, F(X)): \widetilde{f}^{*} N_{P / X \times F(X)} \text { has constant local splitting type } \vec{a}\right\}
\end{aligned}
$$

Since $T$ has only one open set, having constant local splitting type $\vec{a}$ is the same as splitting as above. Thus, $T_{L} F_{\vec{a}}(X)=\operatorname{Mor}_{L}\left(T, F_{\vec{a}}(X)\right)$ is the claimed subspace of $T_{L} F(X)$.

Using the inclusion $T_{L} F(X)=H^{0}\left(N_{L / X}\right) \rightarrow H^{0}\left(\mathcal{O}(1)^{n-1}\right) \cong T_{L} \mathbb{G}(1, n)$, We can represent every $v \in T_{L} F(X)$ by a collection of $n-1$ linear forms $v=$ 
$\left(v_{2}, \ldots, v_{n}\right)$ where $v_{i}\left(x_{0}, x_{1}\right)=v_{i}^{(0)} x_{0}+v_{i}^{(1)} x_{1}$. Such a collection corresponds to the first order deformation

$$
\mathcal{L} \subset T \times X \text { defined by } V\left(x_{2}-\epsilon v_{2}, \ldots, x_{n}-\epsilon v_{n}\right) .
$$

Equivalently, if $a_{i j}$ are affine coordinates on the Grassmannian around the line $L=V\left(x_{2}, \ldots, x_{n}\right)$, i.e.

$$
\left(\begin{array}{llll}
a_{20} & a_{30} & \cdots & a_{n 0} \\
a_{21} & a_{31} & \cdots & a_{n 1}
\end{array}\right) \longleftrightarrow L: x_{i}=a_{i 0} x_{0}+a_{i 1} x_{1}
$$

this corresponds to the morphism $T \rightarrow \mathbb{G}(1, n)$ determined by the map of rings

$$
\mathbb{C}\left[a_{i j}\right] \rightarrow \mathbb{C}[\epsilon] /\left(\epsilon^{2}\right), \quad \text { defined by } a_{i j} \mapsto v_{i}^{(j)} \epsilon .
$$

Given $L \subset X=V(f)$, we have seen that the splitting type of $N_{L / X}$ is determined by the polynomials

$$
f_{i}\left(x_{0}, x_{1}\right):=\left.\frac{\partial f}{\partial x_{i}}\right|_{L} .
$$

We will now see that if $L$ has splitting type $\vec{a}$, then the tangent space to $F_{\vec{a}}(X)$ at $L$ is in turn determined by the $f_{i}\left(x_{0}, x_{1}\right)$ and the higher derivatives

$$
H_{i j}\left(x_{0}, x_{1}\right):=\left.\frac{\partial^{2} f}{\partial x_{i} \partial x_{j}}\right|_{L} .
$$

As always, choose coordinates so that $L=V\left(x_{2}, \ldots, x_{n}\right)$. We can write

$$
\frac{\partial f}{\partial x_{i}}=f_{i}\left(x_{0}, x_{1}\right)+\sum_{j=2}^{n} x_{j} H_{i j}\left(x_{0}, x_{1}\right)+\left(x_{2}, \ldots, x_{n}\right)^{2} .
$$

If $v \in T_{L} F(X) \subset H^{0}\left(\mathcal{O}(1)^{n-1}\right)$, then pulling back the short exact sequence

$$
\left.0 \longrightarrow N_{P / X \times F(X)} \longrightarrow N_{P / \mathbb{P}^{n} \times F(X)} \longrightarrow N_{X \times F(X) / \mathbb{P}^{n} \times F(X)}\right|_{P} \longrightarrow 0
$$

to $\mathcal{L}$, we see that $\mathcal{E}_{v}$ fits into a short exact sequence

$$
0 \longrightarrow \mathcal{E}_{v} \longrightarrow \beta^{*} \mathcal{O}(1)^{n-1} \longrightarrow \beta^{*} \mathcal{O}(d) \longrightarrow 0
$$

where the map on the right is given by the collection of polynomials

$$
\tilde{f}_{i}=\frac{\partial f}{\partial x_{i}}\left(x_{0}, x_{1}, \epsilon v_{2}, \ldots, \epsilon v_{n}\right)=f_{i}\left(x_{0}, x_{1}\right)+\epsilon \sum_{j=2}^{n} v_{j} H_{i j}\left(x_{0}, x_{1}\right) .
$$

The deformation $\mathcal{E}_{v}$ will be trivial - i.e. a direct sum of $\beta^{*} \mathcal{O}\left(a_{i}\right)$ - if and only if the $\tilde{f}_{i}$ satisfy the same syzygies as the $f_{i}\left(x_{0}, x_{1}\right)$, now with coefficients in $\mathbb{C}[\epsilon] /\left(\epsilon^{2}\right)$. To make this precise, suppose that the maps in the normal bundle sequence at our line are given explicitly by

$$
0 \rightarrow N_{L / X} \stackrel{\left(c_{i k}\right)}{\longrightarrow} \mathcal{O}(1)^{n-1} \stackrel{\left(f_{2}, \ldots, f_{n}\right)}{\longrightarrow} \mathcal{O}(d) \rightarrow 0
$$


where the $c_{i k}$ are homogenous polynomials of degree degree $1-a_{k}$ in $x_{0}$ and $x_{1}$ which necessarily satisfy $\sum_{i=2}^{n} c_{i k} f_{i}=0$. Then for $v$ to lie in $T_{L} F_{\vec{a}}(X)$, there must exist $c_{i k}^{\prime}$ of degree $1-a_{k}$ such that

$$
\sum_{i=2}^{n}\left(c_{i k}+\epsilon c_{i k}^{\prime}\right)\left(f_{i}+\epsilon \sum_{j=2}^{n} H_{i j} v_{j}\right)=0
$$

Equivalently, this shows the following.

Lemma 4.2. A tangent vector $v \in T_{L} F(X) \cong H^{0}\left(N_{L / X}\right) \subset H^{0}\left(\mathcal{O}(1)^{n-1}\right)$ is in the subspace $T_{L} F_{\vec{a}}(X)$ if and only if for each $k$,

$$
\sum_{i=2}^{n} \sum_{j=2}^{n} c_{i k} H_{i j} v_{j} \in \operatorname{span}\left\{f_{2}, \ldots, f_{n}\right\} \text { with coefficients of degree } 1-a_{k}
$$

This appears to be a somewhat complicated condition, but with nice choices of the $c_{i k}$ and $f_{i}$, the linear conditions in the lemma become clear. We use this strategy to prove the following.

Theorem 4.3. If $u(\vec{a}) \leq 2 n-d-3$, then $F_{\vec{a}}(X)$ has codimemsion $u(\vec{a})$ for general $X$.

Proof. First note that if $a_{1} \leq-2$, then $u(\vec{a}) \geq h^{0}(\mathcal{O}(\vec{a}))>2 n-d-3$, so we may restrict our attention to lines $L$ with

$$
N_{L / X} \cong \mathcal{O}(-1)^{b} \oplus \mathcal{O}^{\ell} \oplus \mathcal{O}(1)^{m}
$$

Since $h^{1}\left(N_{L / X}\right)=0$, such a line is always a smooth point of $F(X)$ with

$$
\operatorname{dim} T_{L} F(X)=2 n-d-3
$$

The expected codimemension of lines with this splitting type is $b m$, so we can assume $b m \leq 2 n-d-3$. To prove the theorem, it suffices to find $L \subset X$ with this normal bundle where $T_{L} F_{\vec{a}}(X) \subset T_{L} F(X)$ has codimension at least $b m$. Then upper semicontinuity of dimension of fibers together with Theorem 3.1 will imply that $\operatorname{codim} F_{\vec{a}}(X)=u(\vec{a})$ for general $X$.

Now, consider the map

$$
\mathcal{O}(-1)^{b} \oplus \mathcal{O}^{\ell} \oplus \mathcal{O}(1)^{m} \rightarrow \mathcal{O}(1)^{n-1}
$$


given by

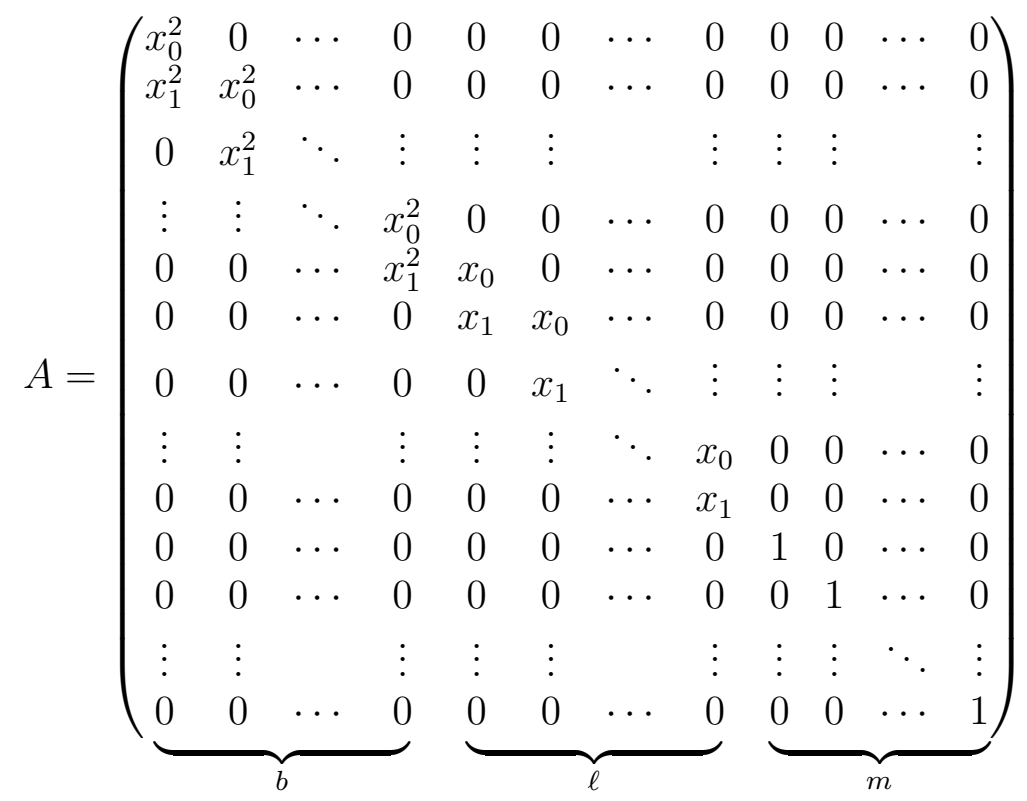

The map to the cokernel of this inclusion is given by the maximal minors of $A$. If $A_{i}$ is $A$ with the $i$ th row deleted, then we have

$$
m_{i}=(-1)^{i} \operatorname{det} A_{i}=(-1)^{i} \begin{cases}x_{0}^{2 i-2} x_{1}^{d-2 i+1} & i \leq b \\ x_{0}^{b+i-1} x_{1}^{d-b-i} & b+1 \leq i \leq b+\ell \\ 0 & b+\ell+1 \leq i \leq n-2 .\end{cases}
$$

Since the $m_{i}$ have no common zeroes, we can apply Bertini's theorem to find some smooth hypersurface $X=V(f)$ containing $L$ such that $f_{i+1}=m_{i}$ are the partial derivatives of $f$ restricted to $L$. For $b+\ell+2 \leq k \leq n-1$, we have $c_{i, k-1}=\delta_{i, k}$, so the condition (4.2) that $v \in T_{L} F_{\vec{a}}(X)$ becomes that the coefficient of $x_{0}^{e} x_{1}^{d-e-1}$ in

$$
\sum_{j=2}^{n} H_{k j}\left(x_{0}, x_{1}\right) v_{j}\left(x_{0}, x_{1}\right)
$$

must vanish for odd $e<2 b$. The general form of an element

$$
v \in T_{L} F(X)=H^{0}\left(N_{L / X}\right) \subset H^{0}\left(\mathcal{O}(1)^{n-1}\right)
$$

is

$$
\begin{array}{r}
v=(\underbrace{0, \ldots, 0}_{j=2, \ldots, b+1}, \underbrace{\lambda_{1} x_{0}, \lambda_{1} x_{1}+\lambda_{2} x_{0}, \ldots, \lambda_{\ell-1} x_{1}+\lambda_{\ell} x_{0}, \lambda_{\ell} x_{1}}_{j=b+2, \ldots, b+\ell+1} \\
\underbrace{\mu_{1}^{(0)} x_{0}+\mu_{1}^{(1)} x_{1}, \ldots, \mu_{m}^{(0)} x_{0}+\mu_{m}^{(1)} x_{1}}_{j=b+\ell+2, \ldots, n-2})
\end{array}
$$


If we can find a smooth $X$ with appropriate $H_{k j}$, then setting the coefficient of $x_{0}^{e} x_{1}^{d-e-1}$ in (4.3) to zero will give an independent linear condition on the coordinates $\lambda_{i}$ and $\mu_{i}^{(j)}$ for each of the $b$ possible values of $e$ and $m$ possible values of $k$, showing that $\operatorname{codim}\left(T_{L} F_{\vec{a}}(X) \subset T_{L} F(X)\right)=b m$. Suppose that

$$
H_{k j}\left(x_{0}, x_{1}\right)= \begin{cases}x_{1}^{d-2}+x_{0}^{3} x_{1}^{d-5} & \text { if } j=k \geq b+\ell+2 \\ x_{0}^{2 i} x_{1}^{d-2 i-2} & \text { if } j-b=i+(k-\ell-b-2)(b-2) \\ 0 & \text { otherwise. }\end{cases}
$$

Above, if the exponent on a variable is negative, it is understood that we omit that term. With this choice, setting the coefficient of $x_{0} x_{1}^{d-2}$ to zero in (4.3) forces each $\mu_{k}^{(0)}=0$. Similarly, if $e=3<2 b$, setting the coefficient of $x_{0}^{3} x_{1}^{d-4}$ to zero forces each $\mu_{k}^{(1)}=0$. Then for the remaining $b-2$ values $e=5, \ldots, 2 b-1$ and each $b+\ell+2 \leq k \leq n-1$, the only contribution to $x_{0}^{e} x_{1}^{d-e-1}$ comes from the

$$
j=b+\frac{e-1}{2}+(k-\ell-b-2)(b-2)
$$

term of the sum in (4.3), when $\lambda_{j} x_{0}$ meets $H_{k j}=x_{0}^{e-1} x_{1}^{d-e-1}$. This gives $m(b-2)$ more conditions $\lambda_{j}=0$. Finally, Bertini's theorem guarantees that there exists a smooth hypersurface with these $f_{i}$ and $H_{k j}$, so we are done.

\section{Important EXAmples}

5.1. Cubic hypersurfaces. As we have seen, there are only two possible splitting types for the normal bundle of a line $L$ on a cubic hypersurface $X$ : either

$$
N_{L / X}=\mathcal{O} \oplus \mathcal{O} \oplus \mathcal{O}(1)^{n-4} \quad \text { (balanced) }
$$

or

$$
N_{L / X}=\mathcal{O}(-1) \oplus \mathcal{O}(1)^{n-3} \quad \text { (unbalanced). }
$$

Since $h^{0}\left(N_{L / X}\right)=2 n-6$ for either splitting type, the Fano scheme is always smooth of that dimension. To simplify notation, let $F^{\prime}=F_{(-1,1, \ldots, 1)}(X)$ be the locus of unbalanced lines. The expected codimension of $F^{\prime}(X)$ is $n-3$.

Specializing our analysis in the previous section gives rise to a concrete description of the tangent space to $F^{\prime}(X)$. Given a line $L \in F^{\prime}(X)$, we can always choose coordinates so that $L=V\left(x_{2}, x_{3}, x_{4}\right)$. Looking at our favorite short exact sequence of normal bundles,

$$
0 \rightarrow N_{L / X} \rightarrow \mathcal{O}(1)^{n-1} \stackrel{\left(f_{2}, \ldots, f_{n}\right)}{\longrightarrow} \mathcal{O}(d) \rightarrow 0
$$

where $f_{i}=\left.\frac{\partial f}{\partial x_{i}}\right|_{L}$, we see that

$$
L \in F^{\prime}(X) \Longleftrightarrow \operatorname{dim} \operatorname{span}\left\{f_{2}, \ldots, f_{n}\right\}=2 .
$$

Given $L \in F^{\prime}(X)$, we can thus choose coordinates so that $\frac{\partial f}{\partial x_{i}}=0$ for all $i \geq 4$. Next, note that the span of two quadratic polynomials with no common zeros 
contains exactly two squares. Hence, we can choose coordinates $x_{0}$ and $x_{1}$ on $L$ so that the two squares in the span of $f_{2}$ and $f_{3}$ are $x_{0}^{2}$ and $x_{1}^{2}$. Finally, after a possible linear change of variables between $x_{2}$ and $x_{3}$, we can write

$$
f=x_{2} x_{0}^{2}+x_{3} x_{1}^{2}+\sum_{i \geq j} h_{i j}\left(x_{0}, x_{1}\right) x_{i} x_{j}+\left(x_{2}, x_{3}, x_{4}\right)^{3} .
$$

With this set up, the tangent space to the Fano scheme is simply

$$
T_{L} F(X)=\left\{v=\left(v_{2}, \ldots, v_{n}\right) \in H^{0}\left(\mathcal{O}(1)^{n-1}\right) \cong T_{L} \mathbb{G}(1, n): v_{2}=v_{3}=0\right\} .
$$

Then Lemma 4.2 tells us that the condition for $v=\left(0,0, v_{4}, \ldots, v_{n}\right)$ to be in the tangent space is that the coefficient of $x_{0} x_{1}$ in $\sum_{j} H_{i j}\left(x_{0}, x_{1}\right) v_{j}\left(x_{0}, x_{1}\right)$ vanishes for each $i \geq 4$. Writing $H_{i j}\left(x_{0}, x_{1}\right)=H_{i j}^{(0)} x_{0}+H_{i j}^{(1)} x_{1}$ and identifying coordinates on $T_{L} F(X)$ as the coefficients $v_{j}^{(0)}$ and $v_{j}^{(1)}$ for $j \geq 4$, we have shown the following.

Lemma 5.1. In our chosen coordinates,

$$
T_{L} F^{\prime}(X)=\operatorname{ker}\left(\begin{array}{ccccc}
H_{44}^{(1)} & H_{44}^{(0)} & \cdots & H_{4 n}^{(1)} & H_{4 n}^{(0)} \\
\vdots & \vdots & \ddots & \vdots & \vdots \\
H_{4 n}^{(1)} & H_{4 n}^{(0)} & \cdots & H_{n n}^{(1)} & H_{n n}^{(0)}
\end{array}\right) \subset T_{L} F(X) .
$$

The codimension of $T_{L} F^{\prime} \subset T_{L} F$ is thus the rank of this matrix.

Because $\Sigma_{(-1,1, \ldots, 1)}$ is smooth, Sard's theorem tells us that $F^{\prime}(X)$ is smooth in general. However, for some $X$, the locus $F^{\prime}(X)$ may be singular, and we can ask: what sorts of singularities occur? We use the above description of the tangent space to $F^{\prime}(X)$ to answer this question for cubic threefolds.

Theorem 5.2. For $X \subset \mathbb{P}^{4}$ a smooth cubic hypersurface, $F^{\prime}=F^{\prime}(X)$ is a curve that has at worst nodes as singularities.

Proof. Suppose $L \in F^{\prime}$ and $\operatorname{dim} T_{L} F^{\prime}=2$. Then $T_{L} F^{\prime}=T_{L} F$, so after choosing coordinates so that $f$ has the form in (5.1), Lemma 5.1 tells us that $h_{44}=0$. Geometrically, this says that the intersection of $X$ with the 2-plane defined by $x_{2}=x_{3}=0$ is $3 L$.

We now use the explicit local equations Example 2.4 to compute the tangent cone to $F^{\prime}$ at $L$. Let $a_{i j}$ be local coordinates on $\mathbb{G}(1,4)$ as in that section. First, note that the Fano scheme $F(X)$ is cut out by 4 polynomials. These polynomials are the coefficients of the polynomial in $x_{0}$ and $x_{1}$ that results from plugging $x_{i}=a_{i 0} x_{0}+a_{i 1} x_{1}$ for $i=2,3,4$ into $f$. With $f$ having the form (5.1), the leading terms of these four polynomials are $a_{20}, a_{21}, a_{30}$, and $a_{31}$, so the tangent plane to the Fano scheme is

$$
T_{L} F(X)=V\left(a_{20}, a_{21}, a_{30}, a_{31}\right) .
$$

Note also that since $h_{44}=0$, the quadratic terms of these four polynomials are in the ideal $I=\left\langle a_{20}, a_{21}, a_{30}, a_{31}\right\rangle$. 
The locus $F^{\prime} \subset \mathbb{G}(1,4)$ is cut out by the equations of $F$ together with the determinant of the matrix.

$$
C(-1)=\left(\begin{array}{lll}
C_{20} & C_{30} & C_{40} \\
C_{21} & C_{31} & C_{41} \\
C_{22} & C_{32} & C_{42}
\end{array}\right)
$$

where $C_{i j}$ are polynomials in $a_{k \ell}$ defined implicitly by

$$
\frac{\partial f}{\partial x_{i}}\left(x_{0}, x_{1}, a_{20} x_{0}+a_{21} x_{1}, a_{30} x_{0}+a_{31} x_{1}, a_{40} x_{0}+a_{41} x_{1}\right)=C_{i 0} x_{1}^{2}+C_{i 1} x_{1} x_{0}+C_{i 2} x_{0}^{2} .
$$

We claim that, modulo the ideal $I$, the leading term of $\operatorname{det} C(-1)$ is a multiple of $a_{40} a_{41}$. First, observe that $C_{22}$ and $C_{30}$ are the only entries that begin with constant terms, the rest being linear or higher order in the $a_{i j}$. Next, we have

$$
\frac{\partial f}{\partial x_{4}}=\lambda x_{4}^{2}+\left(x_{2}, x_{3}\right)
$$

so the linear terms of the entries $C_{4 i}$ in the last column are all in the ideal $I$.

Putting these observations together, we see that the leading term of $\operatorname{det} C(-1)$ modulo $I$ is $C_{41}$ mod $I$, which is $2 \lambda a_{40} b_{41}$. Thus, $I\left(F^{\prime}\right)$ can be generated by five polynomials whose leading terms are $a_{20}, a_{21}, a_{30}, a_{31}$, and $a_{40} a_{41}$. The leading term of any polynomial in $I\left(F^{\prime}\right)$ is therefore in the ideal generated by these leading terms. This shows that the tangent cone to $L \in F^{\prime}$ is a union of the two distinct lines $V\left(a_{40}\right)$ and $V\left(a_{41}\right)$ in the tangent plane, so $L \in F^{\prime}$ must be a node.

5.2. The Fermat quartic threefold. Although we know that $F_{\vec{a}}(X)$ has the expected dimension for general $X$, sometimes $F_{\vec{a}}(X)$ has larger dimension for certain $X$.

One illuminating example of this is when $X=V\left(x_{0}^{4}+\ldots+x_{4}^{4}\right)=V(f) \subset$ $\mathbb{P}^{4}$ is the Fermat quartic threefold. If $H$ is a hyperplane defined by $x_{i}=\zeta x_{j}$ for $\zeta$ a 4 th root of -1 , then the hyperplane section $H \cap X$ is a cone over a smooth quartic curve in $\mathbb{P}^{2}$. We claim that for any line $L$ on this cone, we have $N_{L / X}=\mathcal{O}(-2) \oplus \mathcal{O}(1)$. After possibly permuting coordinates (which preserves the equation of $X$ ), we can assume $L$ has the form

$$
L=\left\{\left[x_{0}, x_{1}, x_{0} a, x_{0} b, x_{1} \zeta\right]:\left[x_{0}, x_{1}\right] \in \mathbb{P}^{1}\right\}
$$

for some $a, b \in \mathbb{C}$ with $a^{4}+b^{4}+1=0$. From this, we see that

$$
\operatorname{span}\left\{\left.\frac{\partial f}{\partial x_{i}}\right|_{L}\right\}=\left\langle x_{0}^{3}, x_{1}^{3}\right\rangle,
$$

which is two-dimensional. In particular, the right hand map in the sequence

$$
0 \longrightarrow N_{L / X} \longrightarrow \mathcal{O}(1)^{3} \longrightarrow \mathcal{O}(4) \longrightarrow 0
$$


is given by a collection of three necessarily linearly dependent polynomials, implying $\mathcal{O}(1)$ includes into its kernel, $N_{L / X}$. It follows by degree considerations that $N_{L / X}=\mathcal{O}(-2) \oplus \mathcal{O}(1)$. Because $h^{0}\left(N_{L / X}\right)=2$, the family of lines on each of these hyperplane sections corresponds to a nonreduced quartic curve on $F(X)$. Since there are 40 such hyperplanes - corresponding to the $\left(\begin{array}{l}5 \\ 2\end{array}\right)$ choices of two coordinates $x_{i}, x_{j}$ and 4 choices of $\zeta$ - computing the class $[F(X)]=c_{5}\left(\operatorname{Sym}^{4} \mathcal{S}^{*}\right)=320 \sigma_{3,2}$ in the Chow ring of the Grassmannian shows that $F(X)$ is exactly the union of these 40 non-reduced curves.

This is important example in that every line on $F(X)$ has normal bundle $\mathcal{O}(-2) \oplus \mathcal{O}(1)$. That is, the underlying sets of $F(X)$ and $F_{(-2,1)}(X)$ are equal. Nevertheless, $F(X)$ and $F_{(-2,1)}(X)$ are not equal as schemes. In fact, $F_{(-2,1)}(X)$ is the reduced subscheme of $F(X)$, as can be seen from computing the tangent space $T_{L} F_{(-2,1)}(X)$ for general $L \in F(X)$. Suppose $L$ has the form in (15.2). Let

$$
w_{2}=x_{2}-a x_{0}, \quad w_{3}=x_{3}-b x_{0}, \quad \text { and } \quad w_{4}=x_{4}-\zeta x_{1} .
$$

Then $L=V\left(w_{2}, w_{3}, w_{4}\right)$, and we can rewrite the defining equation of $X$ in these coordinates as

$$
\begin{aligned}
x_{0}^{4}+x_{1}^{4}+\left(w_{2}+\right. & \left.a x_{0}\right)^{4}+\left(w_{3}+b x_{0}\right)^{4}+\left(w_{4}+\zeta x_{1}\right)^{4} \\
= & 4 w_{2} a^{3} x_{0}^{3}+4 w_{3} b^{3} x_{0}^{3}+4 w_{4} \zeta^{3} x_{1}^{3} \\
& +6 w_{2}^{2} a^{2} x_{0}^{2}+6 w_{3}^{2} b^{2} x_{0}^{2}+6 w_{3}^{2} \zeta^{2} x_{1}^{2}+\left(w_{2}, w_{3}, w_{4}\right)^{3} .
\end{aligned}
$$

This tells us that

$$
T_{L} F(X)=\left\{\left(v_{2}, v_{3}, v_{4}\right): v_{4}=0, a^{3} v_{2}+b^{3} v_{3}=0\right\} .
$$

The inlcusion $N_{L / X} \rightarrow \mathcal{O}(1)^{3}$ can thus be represented by

$$
\left(\begin{array}{cc}
0 & 0 \\
0 & b^{3} \\
0 & -a^{3}
\end{array}\right),
$$

so Lemma 4.2 tells us that $T_{L} F^{\prime}(X) \subset T_{L} F(X)$ is determined by the condition

$$
x_{0}^{2}\left(b^{3} a^{2} v_{2}-a^{3} b^{2} v_{3}\right) \in \operatorname{span}\left\{x_{0}^{3}, x_{1}^{3}\right\} .
$$

This is equivalent to

$$
b^{3} a^{2} v_{2}^{(1)}-a^{3} b^{2} v_{3}^{(1)}=0 .
$$

If $a$ and $b$ are both non-zero, then plugging in $v_{3}^{(1)}=-\frac{a^{3}}{b^{3}} v_{2}^{(1)}$ we find

$$
\begin{aligned}
0=v_{2}^{(1)}\left(b^{3} a^{2}-a^{3} b^{2}\left(-\frac{a^{3}}{b^{3}}\right)\right)=v_{2}^{(1)}\left(\frac{a^{2}}{b}\right)\left(b^{4}+a^{4}\right) & \Rightarrow \quad v_{2}^{(1)}=0 \\
& \Rightarrow \quad v_{3}^{(1)}=0 .
\end{aligned}
$$

Thus, for general lines $L \in F(X)$ we have $\operatorname{dim} T_{L} F^{\prime}(X)=1$, showing that $F^{\prime}(X)$ is reduced. On the other hand, if one of $a$ or $b$ is zero, then $T_{L} F^{\prime}(X)=$ 
$T_{L} F(X)$ because the left-hand side of (5.4) is zero, so the condition is automatically satisfied. These lines $L$ correspond points where two components of $F(X)$ intersect.

\section{The Class of $\overline{F_{\vec{a}}(X)}$}

For general $X$, knowing that $F_{\vec{a}}(X)$ has the expected codimension in $F(X)$ allows us to compute the class of $\overline{F_{\vec{a}}(X)}$ in the Chow ring of $\mathbb{G}(1, n)$. As noted at the beginning of the proof of Theorem 4.3, for general $X, F_{\vec{a}}(X)$ is only nonempty when

$$
\vec{a}=(-1, \ldots,-1,0, \ldots, 0,1, \ldots, 1)=\left(-1^{b}, 0^{\ell}, 1^{m}\right),
$$

where $-b+m=n-d-1$ and $b+\ell+m=n-2$.

Proposition 6.1. Let $\vec{a}=\left(-1^{b}, 0^{\ell}, 1^{m}\right)$ as above. Then for general $X$, the class of $\overline{F_{\vec{a}}(X)}$ in the Chow ring of the Grassmannian is

$$
\left[\overline{F_{\vec{a}}(X)}\right]=[F(X)] \cdot \Delta_{b}^{m}\left(\frac{c(\mathcal{Q})}{c\left(\operatorname{Sym}^{d-1} \mathcal{S}^{*}\right)}\right)=c_{d+1}\left(\operatorname{Sym}^{d} \mathcal{S}^{*}\right) \cdot \Delta_{b}^{m}\left(\frac{c(\mathcal{Q})}{c\left(\operatorname{Sym}^{d-1} \mathcal{S}^{*}\right)}\right) \text {, }
$$

where $\Delta_{b}^{m}$ is defined in (2.8).

Proof. Restricting the vector bundles $\pi_{*} \mathcal{E}(i)$ and $\pi_{*} \mathcal{F}(i)$ from Section 2.1 to the fibers $F(X)$, we may realize $\overline{F_{\vec{a}}(X)}$ as

$$
\overline{F_{\vec{a}}(X)}=M_{r_{-1}(\vec{a})}\left(\left.\pi_{*} \phi(-1)\right|_{F(X)}\right) .
$$

We have

$$
\left.\pi_{*} \mathcal{E}(i)\right|_{F(X)}=\left.\left.\mathcal{Q}\right|_{F(X)} \otimes \operatorname{Sym}^{1+i} \mathcal{S}^{*}\right|_{F(X)} \quad \text { and } \quad \pi_{*} \mathcal{F}(i)=\left.\operatorname{Sym}^{d+i} \mathcal{S}^{*}\right|_{F(X)},
$$

so if $\iota: F(X) \rightarrow \mathbb{G}(1, n)$ denotes the inclusion of $F(X)$ in the Grassmannian, the class of $F_{\vec{a}}(X)$ in the Chow ring of $F(X)$ is

$$
\Delta_{b}^{m}\left(\frac{\iota^{*} c(\mathcal{Q})}{\iota^{*} c\left(\operatorname{Sym}^{d-1} \mathcal{S}^{*}\right)}\right)=\iota^{*} \Delta_{b}^{m}\left(\frac{c(\mathcal{Q})}{c\left(\operatorname{Sym}^{d-1} \mathcal{S}^{*}\right)}\right) .
$$

Finally, the push-pull formula tells us that the pushforward of this class to the Chow ring of the Grassmannian is given by intersecting with $[F(X)]$.

Example 6.2 $(n=d=5)$. For general quintic hypersurfaces $X \subset \mathbb{P}^{5}$, we have $\operatorname{dim} F(X)=2$. The following table lists the possible splitting types for the normal bundle and the expected codimension of lines with that splitting type

$$
\begin{array}{c|c|c|c|c}
\vec{a} & (-3,1,1) & (-2,0,1) & (-1,-1,1) & (-1,0,0) \\
\hline u(\vec{a}) & 4 & 3 & 2 & 0
\end{array}
$$

Thus, Theorem 4.3 says that a general $X$ contains no lines with splitting type $(-3,1,1)$ or $(-2,0,1)$ and finitely many lines with splitting type $(-1,-1,1)$. So we can ask: how many unbalanced lines are there? Since $\Sigma_{(-1,-1,1)}$ is smooth, 
Sard's theorem tells us that $F_{(-1,-1,1)}(X)$ is smooth, and hence reduced, for general $X$. Thus, the answer is the degree of the class given in Proposition 6.1]

$$
\left[F_{(-1,-1,1)}(X)\right]=\left[\overline{F_{(-1,-1,1)}(X)}\right]=c_{6}\left(\operatorname{Sym}^{5} \mathcal{S}^{*}\right) \cdot \Delta_{2}^{1}\left(\frac{c(\mathcal{Q})}{c\left(\operatorname{Sym}^{4} \mathcal{S}^{*}\right)}\right) .
$$

Here, $\Delta_{2}^{1}$ means we need the degree 2 piece of the quotient in the brackets. Writing

$$
\frac{c(\mathcal{Q})}{c\left(\operatorname{Sym}^{4} \mathcal{S}^{*}\right)}=\left(1+\sigma_{1}+\sigma_{2}+\ldots\right)\left(1-\left(c_{1}+c_{2}+\ldots\right)+\left(c_{1}+c_{2}+\ldots\right)^{2}-\ldots\right)
$$

where $c_{i}=c_{i}\left(\operatorname{Sym}^{4} \mathcal{S}^{*}\right)$, we see that the degree 2 part is

$$
c_{1}^{2}+c_{2}+\sigma_{2}-c_{1} \sigma_{1} \text {. }
$$

Using the splitting principle, we calculate

$$
c_{1}=10 \sigma_{1} \quad \text { and } \quad c_{2}=35 \sigma_{1}^{2}+20 \sigma_{1,1}=35 \sigma_{2}+55 \sigma_{1,1},
$$

SO

$$
\Delta_{2}^{1}\left(\frac{c(\mathcal{Q})}{c\left(\operatorname{Sym}^{4} \mathcal{S}^{*}\right)}\right)=100 \sigma_{1}^{2}+35 \sigma_{2}+55 \sigma_{1,1}+\sigma_{2}-10 \sigma_{1}^{2}=126 \sigma_{2}+145 \sigma_{1,1}
$$

Another application of the splitting principle shows that

$$
c_{6}\left(\operatorname{Sym}^{5} \mathcal{S}^{*}\right)=600 \sigma_{1,1} \sigma_{1}^{4}+1450 \sigma_{1,1}^{2} \sigma_{1}^{2}-225 \sigma_{1,1}^{3}=3250 \sigma_{4,2}+2425 \sigma_{3,3} .
$$

Putting this all together, we find

$$
\left[F_{(-1,-1,1)}(X)\right]=\left(126 \sigma_{2}+145 \sigma_{1,1}\right)\left(3250 \sigma_{4,2}+2425 \sigma_{3,3}\right)=761125 \sigma_{4,4}
$$

That is, there are 761125 unbalanced lines on a general quintic fourfold.

\section{Dimension Bounds FOR CERTAin $F_{\vec{a}}(X)$}

In Section 5, we determined the dimension of $F_{\vec{a}}(X)$ for general $X$. In this section, we give upper bounds on the dimension of lines with two particular splitting types which are valid for all smooth hypersurfaces.

7.1. Completely unbalanced lines. There is a unique splitting type $\vec{a}$ which is the "most unbalanced" allowed by the conditions $a_{i} \leq 1$ and $\sum_{i=1}^{n-2}=n-d-1$, namely $\vec{a}=(-d+2,1, \ldots, 1)$. For a given degree $d$, let $F^{\prime}(X)=F_{(-d+2,1, \ldots, 1)}(X)$ denote the locus of lines with this splitting type. The expected codimension of lines with this splitting type is $(n-3)(d-2)$, so Theorem 3.1 tells us

$$
\operatorname{dim} \Sigma_{(-d+2,1, \ldots, 1)}=2 n-d-3+N-(n-3)(d-2) .
$$

It follows that

$$
\operatorname{dim} F^{\prime}(X) \geq 2 n-d-3-(n-3)(d-2)=n-3-(d-3)(n-2),
$$

and if $d \geq 4$, the locus $F^{\prime}(X)$ is empty for general $X$. However, for certain $X$, a priori, $F^{\prime}(X)$ could be as large as $F(X)$, which is in turn only bounded above 
by $\operatorname{dim} T_{L} F(X)=2 n-6$. The following provides an upper bound of half this amount.

Theorem 7.1. If $X \subset \mathbb{P}^{n}$ is a smooth hypersurface of degree $d \geq 3$, then $\operatorname{dim} F^{\prime}(X) \leq n-3$.

Remark. This is proved for cubics in Corollary 7.6 of [2].

Note that for cubics, the lower bound (17.1) then determines the dimension of $F^{\prime}(X)$ exactly. Given that for $d \geq 4$, the lower bound in (7.1) is trivial, one might expect that Theorem 7.1 is far from the truth of what is actually achieved. However, the bound is sharp, as demonstrated by the following proposition.

Proposition 7.2. Let $X=V\left(x_{0}^{d}+\ldots+x_{n}^{d}\right)$ for $d \geq 4$. Then $\operatorname{dim} F^{\prime}(X)=n-3$.

Proof of Theorem 7.1. Suppose that we have some $L \in F^{\prime}(X)$. Choose coordinates so that $L=V\left(x_{2}, \ldots, x_{n}\right)$ and write the defining equation of $X$ as

$$
f=\sum_{i=2}^{n} x_{i} f_{i}\left(x_{0}, x_{1}\right)+\left(x_{2}, \ldots, x_{n}\right)^{2} .
$$

In the short exact sequence of normal bundles

$$
0 \rightarrow \mathcal{O}(-d+2) \oplus \mathcal{O}(1)^{n-3} \rightarrow \mathcal{O}(1)^{n-1} \stackrel{\left(f_{2}, \ldots, f_{n}\right)}{\longrightarrow} \mathcal{O}(d) \rightarrow 0,
$$

each inclusion of $\mathcal{O}(1)$ defines an independent linear relation of $f_{2}, \ldots, f_{n}$. Hence, the span of $f_{2}, \ldots, f_{n}$ is 2-dimensional. The other partial derivatives $\frac{\partial f}{\partial x_{0}}$ and $\frac{\partial f}{\partial x_{1}}$ vanish along $L$, so the Gauss map

$$
\mathscr{G}: X \rightarrow \mathbb{P}^{n *}, \quad p \mapsto\left[\frac{\partial f}{\partial x_{0}}(p), \ldots, \frac{\partial f}{\partial x_{n}}(p)\right]
$$

sends the line $L$ with degree $d-1 \geq 2$ onto a line.

Now consider the incidence correspondence

$$
\Theta=\left\{(p, q, L) \in X \times X \times F^{\prime}(X): p, q \in L, \mathscr{G}(p)=\mathscr{G}(q), p \neq q\right\} .
$$

Let $\pi_{1}: \Theta \rightarrow X$ be projection onto the first factor, and $\pi_{3}: \Theta \rightarrow F^{\prime}(X)$ projection onto the last factor. Every point in $\pi_{1}(\Theta) \subset X$ is a point where the fiber of the Gauss map consists of 2 or more points. Since the Gauss map is generically one-to-one, $\overline{\pi_{1}(\Theta)}$ cannot be all of $X$. Hence,

$$
\operatorname{dim} \pi_{1}(\Theta) \leq \operatorname{dim}(X)-1=n-2 .
$$

Meanwhile, since the Gauss map is finite, the fibers of $\pi_{1}$, which consist of points with the same image under $\mathscr{G}$, must be finite. It follows that $\operatorname{dim} \Theta \leq n-2$ as well. Finally, our work in the previous paragraph shows that the fibers of $\pi_{3}$ are one-dimensional, so we can conclude that $\operatorname{dim} F^{\prime}(X) \leq n-3$. 
Proof of Proposition 7.2. Let $X=V\left(x_{0}^{d}+\ldots+x_{n}^{d}\right)$ and let $H$ be the hyperplane defined by $x_{n}=\zeta x_{n-1}$ where $\zeta^{d}=-1$. Then $H \cap X$ is a cone over the smooth hypersurface $V\left(x_{0}^{d}+\ldots+x_{n-2}^{d}\right) \subset \mathbb{P}^{n-2}$. Let $L \subset X \cap H$ be any line passing through the vertex of this cone. Then any line in the tangent plane along $L$ to $X \cap H$ is on $X \cap H$ to first order. Hence,

$$
\operatorname{dim} T_{L} F(X) \geq \operatorname{dim} \mathbb{G}(1, n-2)=2 n-6 .
$$

For $d \geq 4$, the only way that $h^{0}\left(N_{L / X}\right)$ can be greater than or equal to $2 n-6$ is when $N_{L / X}$ has $n-3$ copies (the maximal number) of $\mathcal{O}(1)$ 's. Thus, all lines on $X \cap H$, which form a family of dimension at least $n-3$, are in $F^{\prime}(X)$. Hence, $\operatorname{dim} F^{\prime}(X) \geq n-3$, and Theorem 7.1 shows that we have equality.

7.2. Almost completely unbalanced lines. A similar trick involving the Gauss map works to find an upper bound on the dimension of the locus of lines whose normal bundle has the form

$$
N_{L / X} \cong \mathcal{O}(-a) \oplus \mathcal{O}(-b) \oplus \mathcal{O}(1)^{n-4}, \quad a+b=d-3 .
$$

Let $G_{a, b}(X)=F_{(-a,-b, 1, \ldots, 1)}(X)$ denote the locus of such lines. The expected codimension of $G_{a, b}(X)$ is

$$
(n-4)(d-3)+\max \{0, a-b-1\},
$$

giving rise to the lower bound on the dimension

$$
\operatorname{dim} G_{a, b}(X) \geq(n-3)-(d-4)(n-3)-\max \{0, a-b-1\} .
$$

Note that for cubics, $a$ and $b$ are both forced to be zero and these are the balanced lines. In this case, this lower bound coincides with the dimension of the tangent space $T_{L} F(X)$, so $\operatorname{dim} G_{0,0}=2 n-6$. For $d \geq 4$, we have the following upper bound on the dimension.

Theorem 7.3. If $d \geq 4$, then $\operatorname{dim} G_{a, b}(X) \leq n-1$ for all smooth hypersurfaces $X \subset \mathbb{P}^{n}$ of degree $d$.

Proof. Suppose that we have some $L \in G_{a, b}(X)$. Choose coordinates so that $L=V\left(x_{2}, \ldots, x_{n}\right)$ and write the defining equation of $X$ as

$$
f=\sum_{i=2}^{n} x_{i} f_{i}\left(x_{0}, x_{1}\right)+\left(x_{2}, \ldots, x_{n}\right)^{2} .
$$

In the short exact sequence of normal bundles

$$
0 \rightarrow \mathcal{O}(-a) \oplus \mathcal{O}(-b) \oplus \mathcal{O}(1)^{n-4} \rightarrow \mathcal{O}(1)^{n-1} \stackrel{\left(f_{2}, \ldots, f_{n}\right)}{\longrightarrow} \mathcal{O}(d) \rightarrow 0
$$

each inclusion of $\mathcal{O}(1)$ defines an independent linear relation of $f_{2}, \ldots, f_{n}$. Hence, the span of $f_{2}, \ldots, f_{n}$ is 3 -dimensional. The other partial derivatives $\frac{\partial f}{\partial x_{0}}$ and $\frac{\partial f}{\partial x_{1}}$ vanish along $L$, so now the Gauss map

$$
\mathscr{G}: X \rightarrow \mathbb{P}^{n *}, \quad p \mapsto\left[\frac{\partial f}{\partial x_{0}}(p), \ldots, \frac{\partial f}{\partial x_{n}}(p)\right]
$$


sends the line $L$ with degree $d-1 \geq 3$ onto a plane curve. Since the genus of a smooth degree $e$ plane curve is $\left(\begin{array}{c}e-1 \\ 2\end{array}\right)>0$ for $e \geq 3$, such a map cannot be an embedding. Hence, there exists some point on $L$ where the differential is zero, or two points on $L$ that have the same image (or both).

First consider the incidence correspondence

$$
\Theta=\left\{(p, L) \in X \times G_{a, b}(X): p \in L \text { and }\left(\left.d \mathscr{G}\right|_{L}\right)_{p}=0\right\}
$$

with projections $\pi_{1}: \Theta \rightarrow X$ and $\pi_{2}: \Theta \rightarrow G_{a, b}(X)$. If $p \in X$ has $\operatorname{dim} \pi_{1}^{-1}(p)=k$, then $\operatorname{dim} \operatorname{ker}(d \mathscr{G})_{p} \geq k$. The dimension of the locus of points in $X$ where the differential of the Gauss map has this rank is at most $n-1-k$. We conclude that $\operatorname{dim} \Theta \leq n-1$, and so $\operatorname{dim} \pi_{2}(\Theta) \leq n-1$ as well.

Next consider the incidence correspondence

$$
\Theta^{\prime}=\left\{(p, q, L) \in X \times X \times G_{a, b}(X): p, q \in L, \mathscr{G}(p)=\mathscr{G}(q), p \neq q\right\} .
$$

Because the image of $\Theta^{\prime}$ onto the first factor consists of points where $\mathscr{G}$ fails to be one-to-one, we must have $\operatorname{dim} \pi_{1}\left(\Theta^{\prime}\right) \leq \operatorname{dim}(X)-1=n-2$. On the other hand, the fact that the Gauss map is finite guarantees that the fibers $\pi_{1}$ are finite, so $\operatorname{dim} \Theta^{\prime} \leq n-2$, and hence $\operatorname{dim} \pi_{3}\left(\Theta^{\prime}\right) \leq n-2$.

The fact that $\left.\mathscr{G}\right|_{L}$ is not an embedding for any $L \in G_{a, b}(X)$ tells us that $G_{a, b}(X)=\pi_{2}(\Theta) \cup \pi_{3}\left(\Theta^{\prime}\right)$. In particular, we have

$$
\operatorname{dim} G_{a, b}(X)=\max \left\{\operatorname{dim} \pi_{2}(\Theta), \operatorname{dim} \pi_{3}\left(\Theta^{\prime}\right)\right\} \leq n-1,
$$

which is the desired result.

\section{REFERENCES}

[1] R. Beheshti, Lines on projective hypersurfaces, J. Riene Angew. Math. 592 (2006), 1-21.

[2] C. H. Clemens, P. A. Griffiths, The intermediate Jacobian of the cubic threefold, Ann. of Math. Second Series, 95:2 (1972), 281-356.

[3] I. Coskun and E. Riedl, Normal bundles of rational curves in projective space, arXiv:1607.06149

[4] D. Eisenbud and J. Harris, $3264 \&$ All That Intersection Theory, Cambridge University Press, 2016.

[5] J. Harris, B. Mazur, R. Pandharipande, Hypersurfaces of low degree, Duke Math. J. 95:1 (1998), 125-160.

[6] E. Riedl and D. Yang, Kontsevich spaces of rational curves on Fano hypersurfaces, 2014, arXiv:1409.3802

Department of Mathematics, Harvard University, One Oxford Street, CamBRIDGE MA 02138

E-mail address: hannahlarson@college.harvard.edu 